\title{
Formas de hacer Historia, 1984-2020: cavilaciones de una observadora participante ${ }^{1}$
}

\section{Ways of making History, 1984-2020: musings of a participating observer}

Hilda Sabato ${ }^{2}$

\section{Resumen:}

Estas páginas proponen un ejercicio de reflexión sobre la Historia como disciplina en la Argentina de las últimas cuatro décadas, período que coincide con los años centrales de la trayectoria de su autora como historiadora. No se trata de una revisión sistemática de ese vastísimo terreno sino de una incursión personal con el propósito de explorar las principales tendencias de la historiografía en la Argentina en tiempos de importantes transformaciones en la dinámica local y global de la disciplina. El artículo se pregunta por los cambios experimentados en los temas y enfoques predominantes en ese campo de saber y por las condiciones materiales e institucionales de esa producción, así como por su relación con las variaciones en el lugar que ocupa el «hacer Historia» en la sociedad contemporánea.

Palabras clave: historia; historiografía argentina; fines siglo XIX/principios siglo XX

\begin{abstract}
:
This article discusses the changes experienced by History as a discipline in Argentina during the last four decades, which were also the core years in the trajectory of the author of these pages as a historian. Rather than a systematic revision of the vast field of History during that period, the author offers an overview of the main tendencies of historiography in Argentina at a time of significant transformations in the local and global disciplinary trends. The article explores the changes and continuities in the dominant themes and perspectives and in the material and institutional conditions of historiographical production, and reflects on how they relate to the place that «doing History» occupies in contemporary societies.
\end{abstract}

Keywords: history; argentine historiography; late nineteenth/early twentieth century

«LHistoria si può veramente deffinire una guerra illustre contro il Tempo, perché togliendoli di mano gl' anni suoi prigionieri, anzi già fatti cadaueri, li richiama in vita, lipassa in rassegna, e li schiera di nuovo in battaglia» (Alessandro Manzoni, I promessi sposi, Introducción)

\footnotetext{
${ }^{1}$ Trabajo recibido el 31/08/2020. Aceptado el 31/10/2020.

${ }^{2}$ Universidad de Buenos Aires. Consejo Nacional de Investigaciones Científicas y Técnicas. Contacto: sabatohilda@gmail.com
} 
El pasado es, por definición, presupuesto indispensable en la labor del historiador. Esa labor está, a su vez, inserta en el tiempo en que le toca vivir y por lo tanto, constituye también materia prima para su propio quehacer. En estas páginas, me propongo un ejercicio en esa dirección, para reflexionar sobre nuestra disciplina, el oficio y sus cultores en las últimas cuatro décadas que son, asimismo, los años centrales de mi propia trayectoria como historiadora. No se trata de una exploración sistemática de ese vastísimo terreno sino apenas de una incursión en una historia que es también la propia y me sugiere revisiones e inspira interrogantes. Me pregunto aquí por los cambios experimentados en los temas y enfoques predominantes en nuestra disciplina y por las condiciones materiales e institucionales de esa producción, así como por su relación con las variaciones en el lugar que ocupa el «hacer Historia» en nuestra sociedad. ${ }^{3}$

\section{El punto de partida}

En la década de 1980, una ola de cuestionamiento y cambio agitó las aguas de la Historia como disciplina. ${ }^{4}$ Originada en los Estados Unidos y varios países de Europa, sus efectos y ramificaciones tuvieron repercusión global. Por entonces, los presupuestos y consensos que habían sostenido la historiografía durante las décadas centrales del siglo XX sufrieron los embates de nuevas corrientes de pensamiento, sintetizadas en la metáfora del «giro lingüístico». En la Argentina, por su parte, esta tormenta tendría un impacto menor y algo tardío, superpuesto a otras transformaciones de efectos más inmediatos y concretos en las condiciones de producción historiográfica. Y mientras es prácticamente imposible fechar con exactitud el inicio de la crisis epistemológica que eclosionó con fuerza a principios de los 80 , en nuestro caso la nueva era tiene un punto de partida preciso: la caída, a fines de 1983, de la dictadura militar y el inicio de la transición democrática.

\footnotetext{
${ }^{3}$ Retomo en este artículo algunas de las reflexiones que volqué de manera muy preliminar en una conferencia pronunciada por invitación de Juan Suriano en la Universidad Nacional de San Martín (Jornadas Estados generales de Historia, 2015) y publicadas en la revista Papeles de Historia del IDAES, 10:18, 2016. Para la preparación de este texto, conté con las recomendaciones bibliográficas y sugerencias inestimables de Claudio Belini, Laura Cucchi, Mirta Z. Lobato, Nora Pagano, Juan Manuel Palacio y Julio Vezub.

${ }^{4}$ En este texto, Historia con mayúscula refiere a la disciplina; reservo la minúscula para la historia como proceso social.
} 
Se inauguró entonces una etapa fundacional en términos institucionales y profesionales. Producto de las políticas represivas de desmantelamiento de la universidad y de otras instancias públicas de creación libre de conocimiento, el campo de la historiografía estaba arrasado. Como las demás áreas de las ciencias sociales y las humanidades, así como otros ámbitos de la cultura, la Historia había sido afectada por esas políticas, cuyo objetivo era terminar con cualquier expresión autónoma de pensamiento, con los mecanismos previamente existentes para su promoción y desarrollo, y sobre todo, con las figuras que podían ser referentes en ese sentido.

En nuestra disciplina, en las décadas anteriores a la de 1970, se habían generado espacios de renovación atentos a la historiografía de punta en otras latitudes y si bien la profesión seguía básicamente en manos de las escuelas más tradicionales, las novedades fueron importantes y dejaron una huella decisiva para lo que vino después. Fueron los años de influencia de los paradigmas estructuralistas y del predominio de la Historia social, no como campo específico de indagación sino como una manera de aproximarse al pasado; así como del acercamiento a las ciencias sociales, especialmente la sociología y la economía, que ofrecían a la Historia modelos de causalidad fuerte y métodos positivos (Halperin Donghi, 1986). Ese mundo dio algunos de los textos que luego serían clásicos de nuestra historiografía y alimentarían a las generaciones siguientes. Pero en el terreno de las instituciones, esos desarrollos todavía débiles quedaron truncos, víctimas de los embates de la dictadura.

En ese marco, 1984 trajo un terremoto institucional e intelectual sobre todo en las humanidades y ciencias sociales, donde hubo -aunque no en todas partes- cambios inmediatos. Empezó una etapa marcada por el ingreso a la universidad de quienes habían (habíamos) estado al margen durante el período anterior, el regreso de muchos exiliados, la normalización del gobierno universitario, la reorganización de carreras, reformas en los planes de estudio, renovación de los planteles docentes, puesta en marcha de la investigación como eje de la actividad universitaria y recomposición del CONICET, sobre todo en estas áreas. Muchos de quienes encabezaron (encabezamos) esos cambios, apostaban (apostábamos) entonces a la afirmación de la Argentina como democracia pluralista, a la refundación de la universidad pública y al fin de las ortodoxias historiográficas. La renovación en la Historia era parte de un movimiento más general de tintes refundacionales, que abarcaba la vida intelectual, cultural y académica, en toda su diversidad. 
A pesar de las restricciones presupuestarias impuestas por una situación económica general muy complicada para la Argentina, el crecimiento del campo académico fue rápido. En el caso de la Historia, los focos de renovación se multiplicaron en las universidades de todo el país, dando lugar a una tendencia expansiva que, a pesar de algunos altibajos temporarios, no ha cesado; diría, por el contrario, que se ha sostenido firme, ya en clave de reproducción normalizada. En sus comienzos, la revolución institucional fue también una empresa intelectual. Se trataba de recuperar el tiempo perdido, introducir los debates historiográficos que solo habían tenido una recepción marginal en la Argentina, promover la actualización y la transformación del campo, impulsar la investigación y creación de conocimiento según los más novedosos protocolos de la disciplina.

En los momentos iniciales, la Historia social operó como faro de orientación para la renovación. Así, la escuela de los Annales y el llamado marxismo cultural inglés, en sus diferentes variantes, fueron sus fuentes principales de inspiración, y entre los locales, los historiadores formados en torno al grupo otrora encabezado por José Luis Romero se convirtieron en referentes insoslayables (Pagano, 2010). No se trató, sin embargo, de retomar sin más la trayectoria trunca que había iniciado la vanguardia de la década del 60, pues desde entonces la Historia social había experimentado cambios importantes y era, además, objeto de duros cuestionamientos que terminaron por quebrar el consenso historiográfico anterior, también en la Argentina.

\section{Combates por la Historia: una crisis epocal}

La disciplina en su conjunto estaba en crisis, en la medida en que la agitación teórica y epistemológica que hacía tiempo sacudía a otros campos de conocimiento había llegado para quedarse. Se abrió entonces un período de controversias, ensayos y experimentaciones. La Historia se fue desgajando del papel central que había ocupado en el pasado en la forja y la legitimación de identidades (sobre todo nacionales, pero también de clase) así como de su pretensión de explicar globalmente el mundo. Sus formulaciones totalizadoras se habían sustentado -decía el editorial de la revista Annales que algo tardíamente diagnosticaba el virajeen el consenso implícito «que fundaba la unidad de lo social identificándolo con lo real» (1988, p. 291). Ese consenso estaba quebrado. No solo 
cayeron los grandes relatos en los que se inscribía la indagación sobre el pasado, sino que se reavivaron discusiones sobre viejos temas pero con nuevas claves: la naturaleza de la producción historiográfica, el estatuto del texto histórico, la posibilidad misma del conocimiento del pasado. ${ }^{5}$

En esos combates por la historia el llamado «giro lingüístico» planteó desafíos fuertes al postular, en sus versiones más radicales, la intradiscursividad de la Historia, que llevó a desarmar nociones tan básicas para nuestro oficio como las de «fuente»y «prueba». En particular, se minaron algunos de los presupuestos centrales de la Historia social, como los de totalidad y determinación social, y al cuestionar cualquier noción de sujeto que implicara unidad, autonomía y acción consciente, se impugnó la idea de actor social -entendido tanto colectiva como individualmente- así como el concepto de experiencia, en tanto instancia clave de la relación entre estructura y conciencia social. De esta manera, la Historia social dejó de ocupar el lugar hegemónico que había conquistado en las décadas anteriores (Sewell, 2005).

Estas discusiones fueron virulentas en el Norte donde, además de la disputa intelectual, se jugaba una pugna por los recursos institucionales. Para entonces, la polémica había llegado a la Argentina como en sordina. Tal vez porque aquí no había combate posible en el plano institucional, pues la Historia social nunca llegó a ser hegemónica. Por el contrario, como ya señalé, ella era -todavía en los 80- bandera de renovación frente al anquilosamiento de las instituciones académicas oficiales. Pero aún más tarde, cuando esa renovación se hizo posible, esta adoptó perfiles que respondían más a los cambios inducidos por la crisis que a la tradición que le había provisto sus banderas (Romero, 1996; Sabato, 2001). Me refiero a la emergencia, como lo señalaba Carlos Altamirano ya en 1990, de «una nueva coyuntura en la práctica historiográfica sin polos hegemónicos en cuanto a las vías, los instrumentos y los objetos que permiten lecturas, de resultados significativos de nuestro pasado» (Altamirano, 1990).

El campo historiográfico argentino en expansión no fue, por lo tanto, demasiado afectado por las confrontaciones que agitaron a otros espacios, aunque no quedó ajeno a sus consecuencias. Mayor impacto tuvieron en ese momento cuestiones más cercanas y en ese sentido, las

\footnotetext{
${ }^{5}$ Existe una vasta bibliografía referida a esta crisis historiográfica y los cambios que siguieron. Aquí mencionaré solo aquellos títulos directamente vinculados con las afirmaciones de este texto. Para una selección en castellano sobre el giro lingüistico ver Palti (1998).
} 
huellas del propio pasado local resultaron decisivas. Los años de muerte vividos luego de la etapa de ilusiones revolucionarias fueron traumáticos en grado sumo. Las certezas anteriores se derrumbaron: ¿Cómo creer en la razón en medio de la sinrazón? ¿Cómo confiar en las teleologías que prometían un futuro liberador? Las preguntas de los historiadores perdieron la seguridad que brindaban las teorías y los modelos vigentes hasta hacía poco tiempo: ¿Hacia dónde y cómo mirar? ¿Qué buscar? La diversidad de respuestas fue la característica más notoria de esos años.

\section{Una profesión}

La multiplicidad de ámbitos institucionales de producción y circulación historiográfica favoreció esa diversidad. El impulso inicial de mediados de los 80 no decayó en la década siguiente, cuando continuó de manera sostenida la expansión de las universidades y, aunque el CONICET tuvo sus altibajos durante la primera mitad de los 90, recuperó luego su dinamismo. A pesar de esos vaivenes, se prosiguió en el camino de la profesionalización asociada a la consolidación de las instituciones estatales dedicadas a la creación y difusión del conocimiento. Hubo una continuación de la incorporación de docentes y reforma de programas, nuevas generaciones formadas y en formación a través de sistemas de becas y proyectos financiados desde el Estado, una afirmación de criterios de calidad compartidos, una proliferación de trabajos que respondían a esos estándares, la multiplicación de revistas especializadas, libros, colecciones para la difusión de la investigación; en suma, decía en 1996 Luis Alberto Romero -uno de los impulsores de la renovación-: «tenemos una profesión» (Romero, 1996, p.102). Señalaba, sin embargo, que junto con ella surgían problemas relacionados con el poder institucional y corporativo, así como con las rigideces en materia laboral que limitaban la incorporación de nuevas generaciones al sistema.

Esta profesionalización o «normalización», como la han llamado algunos, replica un cuadro común a otra latitudes, donde rigen instituciones y prácticas académicas consolidadas, aunque aquí todo sea un poco más precario, menos previsible, y bastante más inestable. El desembarco inicial de un grupo heterogéneo de historiadores en instituciones en plena recomposición llevó de hecho a coincidir en un conjunto de aspiraciones básicas, destinadas a actualizar la disciplina en todas sus facetas y a poner el énfasis en la investigación. Por cierto que ese desiderátum no 
era ajeno al clima político de cambio que vivía el país, donde la aspiración a una reconstrucción democrática resultó, por unos años, hegemónica. De ahí la voluntad compartida por dar forma a mecanismos institucionales para sustentar la conformación de un campo académico y profesional que pudiera revertir la desintegración anterior y se sostuviera en el tiempo.

Esos intentos -como sabemos- fueron bastante exitosos en términos materiales, a la vez que resultaron en una dinámica disciplinar cada vez más compleja. Las pulsiones y los entusiasmos que impulsaron la renovación post-dictadura fueron dando lugar, hacia finales del siglo, a una lógica que respondía a otros mandatos, individuales y colectivos, propios de la producción y reproducción de un campo académico en construcción en un país con escasa tradición en ese sentido. Así, si por un lado el crecimiento rápido dio lugar a la existencia de núcleos y focos diversos y a la pluralidad de liderazgos intelectuales, con la consecuente riqueza de perspectivas y el surgimiento de zonas de controversia; por el otro, las dificultades institucionales que comenzaron a surgir en el marco siempre inestable de la política argentina, alimentaron conductas corporativas y ciertas tendencias conservadoras orientadas a preservar las frágiles posiciones adquiridas, además de las esperables disputas de poder surgidas en ese marco. Nada que sea muy diferente a lo que ocurre en otros campos de actuación profesional.

Por otra parte, en términos sustantivos, Romero se quejaba de la pobreza de debates y de la «ausencia de una imagen general de nuestra historia» (Romero, 1996, p.159). En efecto, uno de los rasgos básicos de la producción de esos años fue que, en sintonía con las tendencias del resto de la historiografía occidental, no desembocó en la propuesta de interpretaciones omnicomprensivas de nuestra historia ni de claves que permitieran explicarlo todo. En cambio, hubo una fragmentación tanto en materia temática como de perspectivas, acorde con el fin de la hegemonía de los grandes relatos y con la crítica a las visiones historiográficas prevalecientes en las décadas anteriores. Esa tendencia no derivó, sin embargo, en una disgregación sin fin de la investigación, sino en una validación de objetos, interrogantes y enfoques diversos que fueron componiendo un paisaje plural. La producción de esos años revela una variedad de focos de interés, más viejos o más nuevos; una inclinación por miradas acotadas a problemas específicos, y una preferencia por el análisis de caso por sobre interpretaciones más abarcadoras o ensayos de mayor alcance. Pero también muestra que esos trabajos puntuales gira- 
ron en torno de núcleos problemáticos más amplios y agendas más ambiciosas, como veremos a continuación. No se trató del tipo de relato que reclamaba Romero, que brindara interpretaciones globales y tuviera eficacia para incidir en las visiones del pasado que circulan en el espacio público, pero comportó un fuerte cuestionamiento a las versiones hasta entonces vigentes de la historia argentina.

\section{Hitos en una agenda plural}

No pretendo aquí hacer un mapa del vasto terreno que cubre la historiografía de las dos décadas finales del siglo XX, sino apuntar a lo que entiendo fueron las líneas de fuerza que imantaron la disciplina y trazaron las directrices de la agenda privilegiada durante esos años.

En primer lugar, pasado el primer momento de recuperación de la Historia social como bandera de la renovación, dos campos se abrieron paso para ocupar posiciones de liderazgo: la Historia intelectual y cultural y la Historia política. Estas dejaron de considerarse ramas arcaicas y menores, relegadas como estaban desde hacía tiempo a los bordes más tradicionales de la disciplina y protagonizaron buena parte de la innovación historiográfica del período (Sabato, 1995).

Bajo la denominación de Historia intelectual y cultural hago referencia a un campo heterogéneo en cuanto a sus enfoques, métodos y objetos específicos pero que remite siempre a la esfera simbólica y de las significaciones. Ideas sistemáticas, pensamiento no formalizado, discursos de distinta índole, lenguajes políticos, imaginarios, ideologías, visiones del mundo, prácticas culturales, intelectuales... la variedad de interrogantes amplió enormemente los alcances de este universo de estudio de límites difusos y cambiantes. Incluyo en este conjunto a la historia conceptual, que ha ido adquiriendo gran relevancia como campo específico y propuesta epistemológica fuerte. En todos estos terrenos, la expansión y la innovación han sido notables, con una producción muy destacada no solo en el ámbito académico sino con proyecciones en el debate público (Altamirano, 1990 y 2005; Palti, 2005; Bruno, 2010, entre otros).

En cuanto a la Historia política, se benefició no solo por la disolución de la hegemonía ejercida por otras ramas sino, también, por la difundida desconfianza en los modelos teleológicos y las explicaciones estructurales, y por el interés creciente por la acción humana y la contin- 
gencia como dimensiones significativas de la interpretación histórica. La interrogación sobre el poder se vio estimulada por los problemas del presente que tuvieron una importancia decisiva a la hora de definir las preguntas; es fácil asociar la renovación de sus problemáticas a los debates propios de los 90 sobre la democracia y sus transiciones (en América Latina, en Europa Oriental), la caída del socialismo real, la revalorización de la ciudadanía y de la sociedad civil, entre otros. El campo no era nuevo, por cierto, pero experimentó un crecimiento y una renovación intelectual muy notables (Alonso, 1998; Halperin Donghi, 2004; Bonaudo, 2006; Sabato, 2007a y 2014; Botana, 2012; Míguez, 2012)

Si es cierto que la Historia intelectual y cultural y la Historia política picaron en punta, no fueron las únicas ramas en expansión, pues la dinámica institucional, académica y política de esas décadas estimuló también otros desarrollos. Al mirar en conjunto este panorama es posible reconocer orientaciones compartidas y resultados que, desde su especificidad, contribuyeron a reformular las visiones más arraigadas de nuestro pasado, abriéndolo a la interrogación.

En ese sentido, al compás de una retracción de la Historia de su papel legitimante en la construcción de identidades nacionales, buena parte de los trabajos se orientaron a deconstruir mitos y memorias más que a construirlos, y a explorar con nuevas preguntas la llamada «historia nacional». ${ }^{6}$ Esa vocación cuestionadora llevó a privilegiar ciertos períodos sobre otros y el largo siglo XIX -período tardo-colonial a 1930cobró centralidad en la agenda. En ese clima, se revisaron e investigaron con originalidad cuestiones tales como los imaginarios sobre el origen de la nación, el proceso revolucionario y de independencia, el federalismo y sus derivas, la formación del estado, la organización territorial, la estructura agraria pampeana, la vida política y sus distintas expresiones, la conformación de actores sociales y políticos, las prácticas culturales y sus cambios, entre otras. Como resultado de ese movimiento, poco ha quedado en pie de la arraigada imagen del XIX como un período de transiciones lineales y progresivas de la sociedad, la economía y las instituciones de Antiguo Régimen a las del estado-nación, el capitalismo, la modernidad y la democracia. Se fueron construyendo, en cambio, visiones alternativas que, más que postular nuevas totalidades, ofrecen pro-

\footnotetext{
${ }^{6}$ Aquí dejo de lado las investigaciones sobre otras regiones del mundo y períodos históricos que también tuvieron un importante desarrollo en estos años.
} 
ductos parciales de investigaciones abiertas a la discusión plural desde el presente.

De esta manera, la historiografía de las décadas de 1980 y 90 no muestra la «soberanía del fragmento» que ven algunos de sus críticos. Tampoco la fidelidad a reglas formales de construcción del conocimiento propias de la disciplina derivó en puro formalismo y en despolitización, como señalan otros (Tobeña, 2015; Zeitler, 2009), aunque puedan señalarse tendencias parciales en ese sentido.

Quisiera apuntar, en cambio, a un rasgo sustantivo de la historiografía de ese período, que la distingue de lo que había antes y de lo que vino después. Me refiero a la manera en que se planteó la relación entre el presente y el pasado y a la convicción de que ese vínculo debía mediatizarse para no subordinar la operación de conocimiento a los mandatos previos del investigador. No se trató de una propuesta original para un problema que desvela a los cultores de la Historia desde siempre, sino de la activación de una convicción que, con distintas formulaciones, ha formado parte de las tradiciones seculares de la historiografía. Si el presente inspira las pasiones y pulsiones del historiador y por lo tanto sus preguntas, se trataba de evitar que ellas impusieran a priori las respuestas. Este mandato compartido reconocía versiones diversas, pero no creo equivocarme al evocar esta cita de Gabrielle Spiegel, de 1990, como representativa de un clima de época vigente en esos años también en la Argentina:

El núcleo ético del compromiso profesional del historiador ha sido siempre la creencia en que su labor ardua, con frecuencia tediosa, produce cierto conocimiento auténtico de lo 'otro' muerto, un conocimiento que se admite está moldeado por las percepciones del historiador y sus sesgos, pero que mantiene cierto grado de autonomía... (Spiegel, 1990)

Con el punto de partida y de llegada del presente, el historiador interroga desde su propio lugar y recurre a restos e indicios de un pasado presupuesto siguiendo los protocolos de la disciplina que, aunque fluidos y disputados, lo orientan en el proceso de conocimiento. La profesión de historiador, decía por entonces Eric Hobsbawm (2000), «es inevitablemente política e ideológica, aunque lo que un historiador dice o no puede no decir depende estrictamente de reglas y convenciones que requieren pruebas y argumentos...» (p.8). En la Argentina de finales del 
siglo XX formulaciones como estas constituían parte del credo compartido, que buscaba evitar la subordinación al presente y ejercer un control epistemológico crítico sobre las orientaciones políticas e ideológicas propias para poder interpelar al pasado en sus propios términos y dar cuenta de lo que tiene de diferente, de verdaderamente «otro». Se trataba de contribuir a la construcción de un saber específico, cuyo producto fuera, asimismo, un insumo para el debate público diferente del generado desde otros espacios de intervención sobre el pasado (Sabato, 2007 b).

\section{Las novedades del siglo}

El panorama de la disciplina ha variado sustantivamente en los últimos veinte años, no solo en nuestro rincón del mundo. Así, en los centros que habían estado a la vanguardia de las disputas historiográficos el cambio fue notable. Cuando parecía que quienes desafiaban el statu quo ante no pararían hasta lograr la hegemonía, hacia la vuelta del siglo se calmaron las aguas sin que se afirmara una nueva ortodoxia. Los cuestionamientos a la historiografía previa tuvieron efectos importantes en la teoría y en la práctica de la disciplina, pero lo que terminó predominando fue un nuevo consenso algo ecléctico y laxo que se apoyaba más en la crítica a lo anterior que en un nuevo paradigma interpretativo y que desembocó en una segmentación de las miradas, una multiplicidad de lenguajes y estrategias de investigación, y la disolución de hegemonías teóricas o metodológicas. Ya en 2005, William Sewell y Geoff Eley, destacados historiadores que desde sus pasos iniciales en la Historia social habían abrazado luego la Historia cultural, publicaron sendos libros donde revisaban la radicalidad de ese viraje y proponían, en diferentes claves y sin renunciar a la pluralidad de enfoques, recuperar la idea de totalidad para analizar el pasado de las sociedades (Sewell, 2005; Eley, 2005).

Por otra parte, a lo largo de estos últimos veinte años, se han propuesto nuevos «giros» -territorial/espacial, global, transnacional, ambiental, para citar solo algunos- pero ninguno de ellos tuvo la radicalidad que había alcanzado el giro lingüístico y sus derivas (Spiegel, 2009; Surkis, 2012; Kaplan, 2017). De todos ellos, el mayor impacto ha sido el que postula la necesidad de una Historia global, en diferentes variantes. Bajo distintas denominaciones (global propiamente dicha, mundial, croisée, transnacional, entre otras), a todas ellas las reúne un denominador común: la crítica a las Historias nacionales, que focalizan su mirada den- 
tro de las fronteras de cada país o de otros espacios sociopolíticos o culturales específicos. Proponen, en cambio, una redefinición de los marcos y escalas espaciales de indagación, movimiento que en sus versiones más militantes se vincula con la necesidad de estar a tono con los procesos de globalización presentes y de convertir a la Historia en un saber «útil» para estos tiempos. ${ }^{7}$

En fecha más reciente, se ha dado a conocer un reclamo que sintoniza con el de la Historia global, pero que pone el foco en la escala temporal para exigir volver a los tiempos largos. The History Manifesto, publicado en 2014 por David Armitage y Jo Guldi, se lamenta por la pérdida de lugar de la Historia en las sociedades contemporáneas, su ausencia total en la formulación de políticas públicas y su incapacidad para incidir sobre los procesos decisorios; acusa a la disciplina de regodearse con las particularidades y los tiempos cortos, y lanza un llamado a retomar la longue durée braudeliana bajo nuevos términos. Esta propuesta circuló rápidamente y desató una cadena de reacciones, de fuertes adhesiones y críticas. En cualquier caso, como intervención académica, es sintomática de un malestar, de una insatisfacción frente a los consensos débiles de finales del siglo pasado y de nuevas búsquedas para recolocar a la disciplina en el centro de la escena pública (Armitage y Guldi, 2016).

\section{El giro argentino}

Un reclamo semejante alcanzó visibilidad en la Argentina, pero sobre otras bases. La trayectoria historiográfica se alineó solo parcialmente con los cambios en otros escenarios y mientras la inercia de la profesión seguía su curso, fueron surgiendo novedades en varios planos, como veremos enseguida. A todo ello no fue ajena, por cierto, la modificación de las condiciones de producción en la disciplina, vinculadas con transformaciones en el panorama político e institucional.

Una primera aproximación en el largo plazo muestra una gran continuidad en términos de profesionalización y extensión del campo académico, potenciada por la expansión de los recursos. Más carreras, posgrados, becas, subsidios, revistas, publicaciones, reuniones. Claro que no siempre más equivale a mejor y es necesario preguntarse por el sen-

\footnotetext{
${ }^{7}$ Un análisis crítico muy agudo sobre la historia global en Serulnikov (2020). Ver, también, Adelman (2017).
} 
tido de esta inversión social y por cuestiones que requieren del análisis y la reflexión colectiva, como las que refieren a los criterios de calidad y evaluación, los perfiles profesionales, las pautas de formación, la distribución de los recursos, y el lugar de la Historia como saber en la sociedad contemporánea, entre otras.

A lo largo de estas dos décadas hubo también vaivenes, altibajos y rupturas en la trayectoria de la disciplina. En particular, no pueden soslayarse los efectos del quiebre social de 2001, momento bisagra que vino a poner en tela de juicio tanto el optimismo político de la década del 80 como el distanciamiento desencantado y para muchos despolitizado de los 90. Pero mientras para los más jóvenes, según he oído una y otra, se trató de «la peor crisis de sus vidas», para muchos de quienes vivimos otras anteriores resultó un nuevo golpe de los tantos que nos habían tocado y no el peor. En mi caso, como muchos de mi generación viví la crisis como fracaso de las expectativas políticas abiertas con la caída de la dictadura en torno a la posibilidad de construir una democracia representativa y pluralista a partir de la instauración del estado de derecho y de la movilización del potencial progresista que suponía inscripto en las mejores tradiciones de nuestra sociedad.

En ese contexto desesperanzado, en que no se vislumbraba otro futuro que no fuera el de la agonía colectiva, la apuesta política del nuevo gobierno peronista elegido en 2003, con Nestor Kirchner a la cabeza, logró recomponer un horizonte de expectativas que inauguró una nueva era. Supo capitalizar la desilusión con el pasado inmediato para poner en marcha un proyecto que buscaba distanciarse de los ideales del 83 y también de las propuestas del propio peronismo en el 90, reavivó algunas de las consignas de los años 70, reinstaló el pasado como terreno de disputas anticipatorias de las del presente y agitó banderas que, si por un lado lograron amplias adhesiones, por el otro también despertaron una multiplicidad de rechazos provenientes de diferentes sectores sociales y campos ideológicos. Más allá de los muy distintos balances que despierta esa experiencia y lo que siguió después, con la derrota electoral del partido que había estado doce años en el poder y el triunfo de una coalición heterogénea que traía otros mandatos, no creo equivocarme al sostener que el contexto político ideológico de estas dos décadas tuvo incidencia en nuestras formas de hacer Historia.

También hubo modificaciones en las condiciones institucionales, con un fortalecimiento sostenido del CONICET como instancia decisiva en la producción y financiación científica en el período 2004-2015, así 
como con la expansión de las universidades en el terreno de la docencia y la transferencia pero un debilitamiento relativo de su incidencia en las políticas de investigación, orientadas de manera creciente por las agencias gubernamentales. La definición de «áreas estratégicas» privilegiadas en la distribución de recursos se inició en ese período y se acentuó con el cambio de gobierno después de 2015, cuando además se restringieron recursos. Esa definición impactó negativamente sobre la disciplina, pues en términos generales, no tenía cabida en las prioridades definidas entonces y que todavía nos rigen, lo que ha traído incertidumbre a la corporación.

No es fácil detectar cómo afectaron estas transformaciones la producción historiográfica y sería forzado establecer relaciones de determinación en ese plano. Pero resulta evidente que hubo, a partir del nuevo siglo, novedades y cambios de dirección en la investigación, que en parte responden a esos contextos pero también a climas culturales más generales así como a las propias dinámicas internas de una disciplina que, más allá de sus peculiaridades locales, responde a tendencias de índole global. Más que intentar identificar esas conexiones, me pregunto a continuación por cuáles fueron las principales tendencias en esa producción a partir del nuevo siglo.

\section{Nuevas orientaciones}

La investigación histórica de las últimas dos décadas no ha hecho sino seguir expandiéndose en diferentes direcciones, con resultados muy heterogéneos. El panorama que emerge es imposible de resumir en pocas páginas, por lo que lejos de intentar ese recorrido me propongo en cambio condensar las novedades más destacadas en torno a dos grandes núcleos.

Un primer rasgo saliente que distingue a esta historiografía es su neta preferencia por la segunda mitad del siglo XX. El interés contemporáneo cada vez mayor por el pasado cercano, vinculado al recalentamiento del presente, encuentra un terreno fértil en la Argentina, donde tantas cuestiones de ese tiempo relativamente próximo resuenan con intensidad en la escena pública. Dos campos específicos sobresalen en ese marco: los estudios sobre el peronismo y la «Historia reciente», convertidos en espacios prolíficos para la investigación histórica. 
No hace veinte años, en una nota publicada en Punto de Vista, Juan Manuel Palacio señalaba la «notoria desatención de la renovación historiográfica» de las décadas previas en materia de peronismo (Palacio, 2002, 40) y postulaba algunas hipótesis sobre el porqué de ese vacío en una disciplina en plena expansión. El panorama cambiaría de manera notable en la década siguiente, en buena medida bajo el estímulo de la resurgida hegemonía que alcanzó el movimiento peronista en la vida política del país. Así, para 2010, el mismo Palacio hablaba de la conformación de un campo en consolidación, centrado en torno al llamado «primer peronismo» (Palacio, 2010). Por su parte, Ranaan Rein (2009) trazaba un «mapa de ese campo» en el que destacaba que «... el foco del debate se trasladó de los sociólogos a los historiadores, de las perspectivas macro a las micro y de lo político a lo social» (p. 137). Desde entonces, los trabajos sobre el tema se han multiplicado, como lo han hecho también sus ámbitos de reproducción académica (ver Gené y Cristiá, 2008; Rein, 2009; Acha y Quiroga, 2009 (a) y (b); Palacio, 2010; Quiroga, 2011; Garzón Rogé, 2019, entre otros). No ha ocurrido, sin embargo, aquello que Rein auguraba hacia el final de su artículo: «definitivamente -escribía- ha llegado el momento de articular las lecciones de esta gran variedad de estudios específicos para formular una renovada síntesis de este fenómeno tan importante que es el peronismo» (Rein, 2009, p.165). Esa síntesis no se ha producido ni está a la vista.

Una segunda novedad de las últimas décadas ha sido el surgimiento y la consolidación de los estudios sobre «historia reciente». En consonancia con la legitimación de la investigación de pasados próximos en la práctica historiográfica en otras latitudes, en la Argentina su afianzamiento se vinculó también con desarrollos locales y en particular, con las formas en que se fue procesando social y políticamente el pasado de la última dictadura militar. De hecho, las investigaciones sobre ese tema constituyen el núcleo central de la expansión de estos estudios, aunque se ha comenzado - muy lentamente- a incorporar otros problemas y períodos (Ver Valobra, 2004/5; Franco y Levin, 2007; Bohoslavsky, 2016; Franco y Lvovich, 2017; Franco, 2018 y 2020, entre otros).

La expansión del campo ha sido explosiva. Una cuidadosa revisión realizada por Marina Franco y Daniel Lvovich da cuenta de la magnitud y las características de esa construcción. Allí también refieren a desafíos y las presiones que plantea el estudio sobre hechos recientes de fuerte carga traumática y con vigencia política e incluso jurídica (Franco y Lvovich, 2017). Desde sus inicios, los esfuerzos por definir un campo histo- 
riográfico dedicado a explorar ese pasado a partir del aparato crítico y los métodos de la disciplina coincidieron con el compromiso de sus impulsores con la causa de los derechos humanos y con los reclamos de «verdad y justicia» en relación con los crímenes de la dictadura. Esa identificación ha sido fundamental para motivar la investigación y para contribuir a su sostenida relación con los temas del debate público, a la vez que ha generado tensiones internas frecuentes. En efecto, con la afirmación de la profesionalización -observa Vera Carnevale (2020)-, más que en otros campos, la Historia reciente «cabalga necesaria u obligadamente sobre la tensión entre el gesto crítico, propio de la disciplina, y el enfoque empático, tributario de la voluntad de intervención política» ( $\mathrm{p}$. 350 ). Ello no ha impedido la consolidación de ese espacio en clave académica, de límites porosos y contactos no siempre fluidos con otras formas de aproximación al pasado reciente que no aspiran al mismo rigor epistemológico.

Además de la preferencia señalada por la historia del siglo $\mathrm{XX}, \mathrm{y}$ en particular por los estudios sobre el peronismo y el pasado reciente, una segunda veta de innovación ha tenido por protagonista al vasto campo de la Historia socio-cultural. La crisis que puso en jaque a la disciplina y dio lugar a la impugnación de la noción de un sujeto privilegiado portador de la historia universal, abrió el camino para la multiplicación de sujetos protagonistas de distintas historias que merecen ser contadas. También cambiaron las formas de entender lo social para incorporar de manera predominante la dimensión cultural. Esta apertura ha dado lugar -no solo en la Argentina- a la expansión de trabajos de investigación dedicados a la identificación y exploración de sujetos colectivos que habían permanecido al margen del interés historiográfico o habían quedado subsumidos en las grandes categorías de nación o clase. Es así que hoy tenemos una variedad de estudios sobre historias de mujeres, pueblos indígenas, afro-descendientes, grupos étnicos y colectividades inmigrantes, sectores subalternos diversos, entre otros, que suelen incorporar análisis social y cultural y crecientemente, perspectivas de género. ${ }^{8}$

Este vasto campo de estudios está tensionado en dos direcciones. Por una parte, y muy en sintonía con el clima de época, se da con fre-

\footnotetext{
${ }^{8}$ La bibliografía sobre estos temas es amplísima. Revisiones del estado de los estudios sobre algunos de esos temas se encuentran, entre otros, en Lobato, 2007 y 2008; Vezub, 2013; Tarquini, 2013; Mandrini, 2015; Tarquini y Casali, 2015; Lamborghini, Geler y Guzmán, 2017; Vezub y de Jong, 2019.
} 
cuencia una empatía explícita con los sujetos colectivos estudiados. Esta inclinación ha dinamizado la investigación a la vez que plantea desafíos a las formas de conocer establecidas y contribuye a enriquecer la disciplina cuyos límites están siempre en disputa. Al mismo tiempo, cuando esa empatía deviene identificación tout court, y se acompaña por el imperativo de reivindicación política, social o cultural, suele inducir a una reificación de los actores esencializando sus identidades y obturando así la posibilidad de interrogación crítica inherente al trabajo del historiador (Vezub, 2013; Lamborghini, Geler y Guzman, 2017).

Un segundo aspecto problemático radica en la inserción de estos estudios en el cuadro más general de la historiografía vigente. Para que la novedad que ha traído esta puesta en escena de sujetos colectivos hasta hace unos años soslayados no se quede apenas en la manifestación testimonial o en el reconocimiento marginal de los actores en cuestión, es fundamental que las investigaciones del campo dialoguen y discutan con el resto, ampliando los registros de análisis de lo social y cultural a otras dimensiones como la política, entre otras. También, que los demás campos registren el impacto de esta producción e incorporen de manera sustantiva lo que ella ha venido a decir y ofrecer en la exploración del pasado (Tarquini y Casali, 2015; Lobato, 2008).

\section{El pasado}

Más allá de estas novedades, la historiografía argentina ha seguido produciendo de manera sostenida en muy diversos campos, con diferentes enfoques y perspectivas. En ese sentido, se observa una continuidad con las décadas anteriores pues la dinámica general de la profesión no ha variado demasiado. Pero al mismo tiempo, nuevas preocupaciones atraviesan la disciplina, que pueden leerse como una reacción ante las propuestas previas de vinculación con el pasado.

Hay, entiendo, una insatisfacción de los historiadores con el lugar que tiene el «hacer Historia» en las sociedades contemporáneas. Desgajada de los imperativos identitarios que llevaron a la disciplina a un lugar de prestigio y poder en la era de las naciones, la historia de finales del siglo XX parecía cobrar autonomía como disciplina y reclamaba el estatuto de un saber desprendido de esos mandatos extraños a sus propios protocolos. Son esa autonomía y ajenidad las que hoy están puestas en cuestión, quizá porque se las asocia con la pérdida de poder y centralidad 
pública de la Historia en estos tiempos. Y si bien en la Argentina las demandas en pos del enfoque global o de una recuperación del tiempo largo no encontraron demasiado eco, surgieron en cambio otros reclamos.

Ante el cambio drástico del horizonte de expectativas que se produjo después de 2001, las certidumbres anteriores fueron prontamente cuestionadas y a ello no escapó el consenso historiográfico de la renovación, que si bien nunca fue homogéneo, había logrado instalarse, a partir de los tardíos 80, como piso compartido para el desarrollo de la profesión. Se ha cuestionado la presunta «despolitización» de la práctica historiográfica, que habría promovido un divorcio entre presente y pasado; la segmentación de temas y problemas; la falta de macro-relatos o interpretaciones globales que dieran sentido al conjunto; la reticencia a visitar el pasado según los sentidos y mandatos impuestos desde el presente, y un supuesto desinterés por la intervención en debates públicos (Adamovsky, 2001; Zeitler, 2009; Tobeña, 2015; Bohoslavsky, 2016; entre otros).

A lo largo de estas páginas, hemos visto que no fue la despolitización ni la soberanía del fragmento lo que caracterizó aquella producción. Tampoco lo fue la indiferencia respecto de la difusión (Sabato, 2007b; Suriano, 2015). En cambio, una clave se encuentra en la forma de entender la relación entre pasado y presente, pues mientras entonces se partía de una problematización de ese vínculo que apuntaba a aplicar con rigor los instrumentos de la crítica para no anticipar, torsionar o forzar resultados en función de las inclinaciones y pasiones previas del historiador, en las últimas décadas han surgido otras propuestas que por el contrario privilegian el anclaje determinante en el presente. A estas tendencias contribuyó seguramente la apelación recurrente al uso político del pasado tanto de la mano del peronismo kirchnerista - primero desde el poder y luego desde la oposición -como de movimientos sociales y militantes de minorías discriminadas en su necesidad de fundar genealogías y de poner en circulación memorias colectivas reafirmantes de su identidad y de su lugar en la historia.

Parte de los cambios que observamos en la historiografía actual responden a esa diversificación en las maneras de entender la relación con el pasado y por lo tanto de hacer Historia. Si bien no hay uniformidad en ese sentido, estas últimas décadas han traído cambios en las temáticas y perspectivas historiográficas impulsados por las nuevas generaciones que hoy hegemonizan el campo académico. Así, las preguntas 
dominantes ya no apuntan, como ocurría con las historias sobre el siglo XIX, a la trabajosa construcción de imágenes alternativas plurales a los mitos fundacionales y los grandes relatos. Esa manera de pensar la historia no se agotó, sigue vigente, pero ha perdido protagonismo. En cambio, nuevos territorios se abrieron a la indagación, en buena medida tributarios de las pulsiones por anudar más estrecha y explícitamente pasado y presente y por revalorizar el uso de la investigación histórica en función de los intereses políticos e ideológicos actuales. Y en ese marco se han producido importantes novedades historiográficas.

Entretanto, otros reclamos recientes no se han traducido en cambios concretos. Así, por ejemplo, no se observa un retorno triunfal de los macro-relatos y las interpretaciones omnicomprensivas, mientras la investigación sigue siendo fragmentada y fragmentaria, diversificada en cuanto a temas y enfoques y a la vez unificada en torno a los protocolos académicos que marcan el paso de cargos, becas y publicaciones con más regulaciones burocráticas que nunca. Tampoco la aspiración de llegar desde la disciplina al gran público ha ido muy lejos, o al menos no tan lejos como para conquistar un lugar de enunciación expectable en el espacio público, que sigue hegemonizado por relatos provenientes de otros ámbitos.

Hasta aquí llega este balance parcial. Con las limitaciones de una observadora que ha sido también participante activa de esta historia, he tratado de distinguir procesos y tendencias, controversias e interrogantes a lo largo de casi cuatro décadas que marcan la reconstrucción de la Historia como disciplina de conocimiento en la Argentina. Habrá que ver cómo sigue a partir de ahora esta historia de la Historia, cuando se ha iniciado una nueva y profunda crisis global que sin duda afectará nuestro quehacer. Estamos, una vez más, en manos a esa constante clave que surca la historia humana, la incertidumbre.

\section{Referencias bibliográficas}

Acha, O. y Quiroga, N. (2009). La normalización del primer peronismo en la historiografía argentina reciente. Estudios Interdisciplinarios de América Latina y el Caribe, 20,2.

Acha, O. y Quiroga, N. (2009). Pliegues de la normalización de los estudios sobre el primer peronismo. En: Rein, R. et al. Los estudios sobre el primer peronismo. Aproximaciones desde el siglo XXI. La Pla- 
ta, Argentina: Archivo Histórico de la Provincia de Buenos Aires. Adamovsky, E. (ed.). (2001). Historia y sentido. Exploraciones en teoría historiográfica. Buenos Aires, Argentina: El Cielo por Asalto.

Adelman, J. (2017). Is global history still possible or has it had its moments?. Aeon Newsletter; https://aeon.co/essays/is-global-history-still-possible-or-has-it-had-its-moment

Alonso, P. (1998). La reciente historia política en la Argentina del ochenta al centenario. Amuario IEHS, 13.

Altamirano, C. (1990).Breve apología de la historia intelectual. Espacios, $8 / 9$.

Altamirano, C. (2006). Para un programa de historia intelectual y otros ensayos. Buenos Aires, Argentina: Siglo XXI.

Armitage, D. y Guldi, J. (2016 [2014]). Manifiesto por la Historia. Madrid, España: Alianza.

Bohoslavsky, E. (2016). Cambios en la historiografía académica en Argentina (2001-2915). História da historiografía, 20.

Bonaudo, M. (2006). La restitución de lo político. Mirando a los historiadores reflexionar sobre la construcción de la comunidad política en la República Posible (Argentina, 1856-1912). L'Ordinaire Latino-Américain, 203.

Botana, N. (2012). Post scriptum: Primera parte (1994)-Segunda parte (2012). En: El orden conservador. La politica argentina entre 1880 y 1916. Buenos Aires, Argentina: Edhasa.

Bruno, P. (2010). Notas sobre la historia intelectual argentina entre 1983 la actualidad. Cercles. Revista d'Història Cultural, 13.

Carnovale, V. (2020). La violencia revolucionaria ante la justicia: nuevos problemas y desafíos historiográficos. PolHis, 25 (13).

Cattaruzza, A. (2008). Los años 60 y 70 en la historiografía argentina (1983-2008). Nuevo mundo, mundos nuevos, 8.

Devoto, F. (Dir.). (2010). Historiadores, ensayistas y gran público. La historiografía argentina en los últimos 20 años (1990-2010). Buenos Aires, Argentina: Biblos.

Eley, G. (2005). A Crooked Line. From cultural history to the history of society. Ann Arbor, Estados Unidos: The University of Michigan Press. 
Franco, M. (2018). La última dictadura argentina en el centro de los debates y las tensiones historiográficas recientes. Tempo e argumento, 10, 23.

Franco, M. y Levín, F. (2007). Historia reciente. Perspectivas y desafíos para un campo en construcción. Buenos Aires, Argentina: Paidós.

Franco, M. y Lvovich, D. (2017). Historia reciente: apuntes sobre un campo de investigación en expansión. Boletín del Instituto de Historia Argentina y Americana «Dr. Emilio Ravignani», Tercera serie, 47.

Garzón Rogé, M. (2019). De enigma a paradoja. Reensamblar la politica de los primeros peronistas (1945-1955). Boletín del Instituto de Historia Argentina y Americana «Dr. Emilio Ravignani», Tercera serie, 51.

Halperin Donghi, T. (1986). Un cuarto de siglo de historiografía argentina, 1960-1985. Desarrollo Económico, 100.

Halperin Donghi, T. (2004). El resurgimiento de la política: problemas y perspectivas. En: Bragoni, B. Microanálisis. Buenos Aires, Argentina: Prometeo.

Hobsbawm, E. (2000). Cuando la pasión ciega a la historia. Clarin, 2 de abril.

Kaplan, T. P. (2017). History and Theory. Writing Modern European Histories after the Linguistic Turn. En: Meng, M. y Seipp, A. Modern Germany in Transatlantic Perspective. Nueva York, Estados Unidos: Berghahn.

Lamborghini, E.; Geler, L. y Guzmán, F. (2017). Los estudios afrodescendientes en Argentina: nuevas perspectivas y desafíos en un país 'sin razas'». Tabula rasa, 27.

Lobato, M. (2007). Niveles y dimensiones de análisis en el mundo del trabajo: notas a partir de una experiencia de investigación. Anuario IEHS, 22.

Lobato, M. (2008). Trabajo, cultura y poder: dilemas historiográficos y estudios de género en Argentina. Estudios de Filosofía Politica e Historia de las Ideas, 9,10.

Mandrini, R. (2015). Historia e historiografía sobre los pueblos indígenas: entrevista con Raúl J. Mandrini (a cargo de María Cristina Bohn Martins). História Unisinos, 19, 1. 
Míguez, E. (2012).Gestación, auge y crisis del orden político oligárquico en la Argentina. Balance de la historiografía reciente. PolHis, 9.

Pagano, N. (2010). La producción historiográfica reciente: continuidades, innovaciones, diagnósticos. En: Devoto, F. (Dir.) (2010). Historiadores, ensayistas y gran público. La historiografia argentina en los últimos 20 años (1990-2010). Buenos Aires, Argentina: Biblos.

Palacio, J. M. (2002). Una deriva necesaria. Notas sobre la historiografía argentina de las últimas décadas. Punto de Vista, 74.

Palacio, J. M. (2010). El primer peronismo en la historiografía reciente: nuevas perspectivas de análisis. Iberoamericana, 39.

Palti, E. (1998). Giro lingüístico e historia intelectual. Bernal, Argentina: Universidad Nacional de Quilmes.

Palti, E. (2005). De la historia de las «ideas» a la historia de los «lenguajes políticos». Las escuelas recientes de análisis conceptual. El panorama latinoamericano. Anales, 7/8.

Quarleri, L. y Lazzari, A. (Coords.). (2015). Dossier «Pueblos indígenas y Antropología en la Argentina. Balances y perspectivas (19842014). Papeles de Trabajo, 9,16.

Quiroga, N. (2011). El partido político en los estudios sobre el primer peronismo. Anuario IEHS, 26.

Rein, R. (2009). De los grandes relatos a los estudios de «pequeña escala»: algunas notas acerca de la historiografía del primer peronismo. En: Rein, R. et al. Los estudios sobve el primer peronismo. Aproximaciones desde el siglo XXI. La Plata, Argentina: Archivo Histórico de la Provincia de Buenos Aires.

Romero, L. A. (1996). La historiografía argentina en la democracia: los problemas de la construcción de un campo profesional. Entrepasados, 10 .

Sabato, H. (1995). Historia política, historia intelectual: viejos temas, nuevas ópticas». En Palacios, M. (Comp.). Siete ensayos de historiografía. España, Argentina. México. Bogotá, Colombia: Editorial de la Universidad Nacional.

Sabato, H. (2001). La historia en fragmentos: fragmentos para una historia. Punto de Vista, 70.

Sabato, H. (2007a). La política argentina en el siglo XIX. Notas sobre una historia renovada. En Palacios, G. (Coord.). Ensayos sobre la 
nueva historia politica de América Latina, s. XIX. México, México: El Colegio de México

Sabato, H. (2007b). Saberes y pasiones del historiador. Apuntes en primera persona. En Marina Franco y Florencia Levin (Comps.). Historia reciente. Perspectivas y desafíos para un campo en construcción. Buenos Aires, Argentina: Paidós.

Sabato, H. (2014). Los desafíos de la república. Notas sobre la política argentina pos Caseros. Estudios Sociales, 46.

Salomón Tarquini, C. (2013). Indígenas e identidades políticas en Argentina (fines del siglo XIX y siglo XX): problemas y perspectivas. Boletín Americanista, año LXIII, 1,66.

Salomón Tarquini, C. y Casali, R. (2015). Los pueblos indígenas de Pampa y Patagonia, siglos XVIII-XX. Un breve estado de las investigaciones. Papeles de Trabajo, 9, 16.

Serulnikov, S. (2020). El secreto del mundo: sobre historias globales y locales en América Latina. História da Historiografia, 13,32.

Sewell, W. H. (2005). Logics of History: Social Theory and Social Transformation. Chicago, Estados Unidos: The University of Chicago Press.

Spiegel, G. (1990). History, Historicism, and the Social Logic of the Text in the Middle Ages. Speculum, 65.

Spiegel, G. (2009). The Task of the Historian Presidential Address, American Historical Review, 114, 1.

Surkis, J. (2012). When Was the Linguistic Turn? A Genealogy, American Historical Review, 117, 3.

Suriano, J. (2015). El Bicentenario de la Revolución de Mayo y los discursos públicos sobre la historia. Tarea. 2,2.

Tobeña, V. (2015). La historia argentina al banquillo. Sobre los usos políticos del pasado y los regímenes de historicidad. Estudios sociológicos, XXXIII, 97.

Vezub, J. E. (2013). El proceso de popularización indígena-criollo en Pampa y Patagonia del siglo XIX. En: Fradkin, R. y Di Meglio, G. (comps.). Hacer politica. La participación popular en el siglo XIX rioplatense. Buenos Aires, Argentina: Prometeo.

Vezub, J. y De Jong, I. (2019). El giro escritural de la historiografía mapuche: alfabeto y archivos en la frontera. Un estado de la cuestión. Quinto Sol, 23, 3. 
Zeitler, E. (2009). El campo historiográfico argentino en la democracia. Transición, profesionalización y renovación. Estudios Históricos, 3. 